\title{
A Raid-Type War-Game Model Based on a Discrete Multi-Weapon Lanchester's Law
}

\author{
Seung-won Baik* \\ Department of Weapons and Mechanical Eng., Korea Military Academy
}

(Received: October 17, 2013 / Revised: October 24, 2013 / Accepted: October 29, 2013)

\begin{abstract}
We propose a war-game model that is appropriate for a raid-type warfare in which, a priori, the maneuver of the attacker is relatively certain. The model is based on a multi-weapon extention of the Lanchester's law. Instead of a continuous time dynamic game with the differential equations from the Lanchester's law, however, we adopt a multiperiod model relying on a time-discretization of the Lanchester's law. Despite the obvious limitation that two players make a move only on the discrete time epochs, the pragmatic model has a manifold justification. The existence of an equilibrium is readily established by its equivalence to a finite zero-sum game, the existence of whose equilibrium is, in turn, well-known to be no other than the LP-duality. It implies then that the war-game model dictates optimal strategies for both players under the assumption that any strategy choice of each player will be responded by a best strategy of her opponent. The model, therefore, provides a sound ground for finding an efficient reinforcement of a defense system that guarantees peaceful equilibria.
\end{abstract}

Keywords: Military Modeling, Game Theory, Heuristic, $\varepsilon$-Equilibrium

* Corresponding Author, E-mail: bsw853@hanmail.net

\section{INTRODUCTION}

A war-game is often an only means to improve a military doctrine or to make an important military decision, such as the reinforcement or reallocation of a weapon system that is supposed to maintain a peaceful equilibrium of a territorial area in which an armed conflict between two nations is likely to bring a grave consequence. An ideal war-game model, therefore, should be able not only to formulate the warfare with an appropriate game theoretic consideration but also to compute an equilibrium in a computation time small enough for a fast-evolving environment of the warfare.

In practice, true equilibria are behind the veil of experimental outcomes from the war-game simulators in which the combat engagement module depends on a human intervention, or the agent strategies determined by a rather arbitrary penalty functions. They are far from a provider of an optimal strategy or equilibrium analysis for general parameters of warfare. The gap is mainly due to the complexity of the cause and effects that determine the course of warfare. We propose a mathematical war-game model that describes a raid of an attacker from a marshaling area to a target area of a defender's territory in which the maneuver of attacker tend to adopt a shortest path and hence is, a priori, relatively certain.

Our war-game model is based on a multi-weapon extention of the Lanchester's law so that the attrition or incapacitation rate of a weapon $i$ is determined by a weighted sum of the numbers of weapons $j$ firing at the weapon, in which each weight is, as in the Lanchester's law, offensive firepower of $j$ with respective to $i$. Instead of a continuous time dynamic game with the differential equations from the Lanchester's law, however, we adopt a multi-period model relying on a time-discretization of the Lanchester's law: the time horizon $[0, T]$ is divided into the time periods of a predetermined length $\Delta$ only on whose time epochs $0, \Delta, 2 \Delta, 3 \Delta, \cdots$, we assume, two 
players make move.

Despite the obvious limitation, the pragmatic model has a manifold justification. The existence of an equilibrium is readily established by its equivalence to a finite zero-sum game, the existence of whose equilibrium is, in turn, well-known to be no other than the LP-duality. It implies then that the war-game model dictates optimal strategies for both players under the assumption that any strategy choice of each player will be responded by a best strategy of her opponent. The model, therefore, provides a sound ground for finding an efficient reinforcement of a defense system that guarantees peaceful equilibria.

Since Lanchester's law is consisted of ally and enemy's homogeneous weapon system and the attrition rate is limited to constant, many studies are still in progress with extention of this model.

Kawaka dealt with allocating fire issue in battle between groups that is consisted of support unit(mainly artillery) and primary unit(mainly infantry), and figured out concentrated firepower to opponent's primary unit is optimal strategy (Kawara, 1973). Later on, Hsiao and Guu applied this model to air-land operations (Hsiao and Guu, 2004). Study of battles between homogeneous weapon systems and heterogeneous weapon systems has continued (Kaup et al., 2005; Roberts and Conolly, 1992; Taylor, 1973). Until now, Colegrave's study is the only one that deals with engagement circumstances which has multiple decision-making elements of weapon systems (Colegrave and Hyde, 1993).

\section{THE MODEL}

Although the proposed model is independent of a particular scenario of warfare, we present one for an intuition of the readers.

\subsection{Scenario}

An attacker makes a raid on an island of the defender's territorial sea attempting to maximize the number of landing crafts with the support from a weapon system of, for instance, battle ships, long-range guns, etc. The defender tries to minimize the opponent's landing crafts by employing an area defense weapon system.

More specifically, each of two parties decides, continuously or discretely, the number of each weapon of her system to fire at each weapon of her counterpart. The objective is to maximize or minimize a linear combination of survived weapons of two systems. We assume naturally that the payoffs of two parties are zero-sum.

\subsection{Notations}

$<$ Parameters $>$

- $i \in\{1, \cdots, m\}$ : Index set of weapons in the defender's weapon system,
- $j \in\{1, \cdots, n\}$ : Index set of weapons in the attacker's weapon system,

- $t \in[0, T]$ : Time epochs in the duration $[0, T]$ of a combat,

- $p_{i j}(t)$ : Offensive firepower at time epoch $t$ of the defender's weapon $i$ to the attacker's weapon $j$,

- $q_{i j}(t)$ : Offensive firepower at time epoch $t$ of the attacker's weapon $j$ to the defender's weapon $i$.

$<$ Decision Variables $>$

- $w_{i}(t)$ : Available number of weapon $i$ of the defender's weapon system at time epoch $t$,

- $z_{j}(t)$ : Available number of weapon $i$ of the attacker's weapon system at time epoch $t$,

- $x_{i j}(t)$ : Number of units of the defender's weapon $i$ that fires at the attacker's weapon $j$ at time epoch $t$,

- $y_{i j}(t)$ : Number of units of the attacker's weapon $j$ that fires at the defender's weapon $i$ at time epoch $t$.

\subsection{Assumption}

In modeling our problem, we assume the followings.

$A_{1}$ : The maneuver of the attacker's weapon system is predictable a priori. Accordingly, the maneuver of the defender weapon system is determined. For instance, the attacker's landing force, due to the nature of a raid, chooses a shortest path from the marshaling area to the target area. Then the defender's battle ships approach, follow, fire at the attacker's mobile force in the territorial sea according to the military doctrine.

$$
\frac{d w_{i}(t)}{d t}=-q_{i j}(t) y_{i j}(t)
$$

$A_{2}$ : The instant rate of attrition or incapacitation a weapon $i$ due to firing of weapon $j$ at a time epoch $t$ is determined by the offensive firepower coefficient $q_{i j}(t)$ of $j$ with respective to $i$ times $y_{i j}(t)$ the number of weapon $j$ participating in firing at weapon $i$ :

$$
\frac{d w_{i}(t)}{d t}=-\sum_{j=1}^{n} q_{i j}(t) y_{i j}(t), \quad \forall i \in\{1, \cdots, m\}, \quad \forall t \in[0, T] .
$$

$A_{3}$ : The losses in fighting forces are reflected in realtime basis.

The offensive power coefficient $q_{i j}(\mathrm{t})$ of $j$ with respective to $i$ in Assumption $A_{2}$ is, in practice, the inverse of the staying power of $i$ with respective to $j$, the number of enemy weapon $j$, at its proper firing level, required to completely incapacitate a unit of weapon $i$. The readers are referred, for instance, to (Marin, 1989; Welr, 1977) for the study of staying power of some pairs of weapons. We note instead that besides other factors the physical distance between two weapons also affects the staying power. From Assumption $A_{1}$ the distances between the 
weapons are known for each time epoch, hence their offensive power coefficients $q_{i j}(\mathrm{t})$ may be assume to be a function of time $t$.

\subsection{Multi-Weapon Lanchester's Law}

Each player decides continuously how many units of each weapon of her system to fire at each weapon of the other's system at each time epoch $t: x_{i j}(t)$ and $y_{i j}(t)$, for $i \in\{1, \cdots, m\}$ and $j \in\{1, \cdots, n\}$. Then the constraints of this particular war-game can be formulated as follows:

$$
\begin{array}{lll}
\sum_{j=1}^{n} x_{i j}(t)=w_{i}(t), & \forall i \in\{1, \cdots, m\}, & \forall t \in[0, T], \\
\frac{d w_{i}(t)}{d t}=-\sum_{j=1}^{n} q_{i j}(t) y_{i j}(t), & \forall i \in\{1, \cdots, m\}, & \forall t \in[0, T], \\
\sum_{i=1}^{m} y_{i j}(t)=z_{j}(t), & \forall j \in\{1, \cdots, n\}, & \forall t \in[0, T], \\
\frac{d z_{j}(t)}{d t}=-\sum_{i=1}^{m} p_{i j}(t) x_{i j}(t), & \forall j \in\{1, \cdots, n\}, & \forall t \in[0, T], \\
x_{i j}(t), y_{i j}(t) \geq 0, & \forall i, \forall j, \forall t . &
\end{array}
$$

The constraints mean that once two players have decided $x_{i j}(t)$ and $y_{i j}(t)$ for all $i, j$, and $t$, the changes in the strength of forces of two parties obeys the multiweapon Lanchester's law under Assumption $A_{2}$.

\subsection{War-Game Model Based on Discrete Time}

Consider a partition of the time interval $[0, T]$ into $N$ periods of length $\Delta,[0, \Delta],[\Delta, 2 \Delta], \cdots,[(t-1) \Delta$, $t \Delta], \cdots,[(N-1) \Delta, N \Delta]$, where $T=N \Delta$.

From the second constraint of section 2.4, the total attrition in weapon system $i$ during $[(t-1) \Delta, t \Delta]$ is,

$$
\begin{aligned}
w_{i}(t \Delta)-w_{i}((t-1) \Delta) & =\int_{(t-1) \Delta}^{t \Delta} \frac{d w_{i}(s)}{d s} d s \\
& =-\sum_{j=1}^{n} \int_{(t-1) \Delta}^{t \Delta} q_{i j}(s) y_{i j}(s) d s .
\end{aligned}
$$

We assume, here, that the offensive firepower $q_{i j}(s)$ is a constant $q_{i j}^{t}$ over the $t$-th period $[(t-1) \Delta, t \Delta]$. The offensive strategy $y_{i j}(s)$ is also assumed to be fixed to a value $y_{i j}^{t}$ over $[(t-1) \Delta, t \Delta]$. Then, from (1) we get,

$$
w_{i}(t \Delta)-w_{i}((t-1) \Delta)=-\sum_{j=1}^{n} \int_{(t-1) \Delta}^{t \Delta} q_{i j}^{t} y_{i j}^{t} \Delta .
$$

Denoting $Q_{i j}^{t}=q_{i j}^{t} \Delta$, we get

$$
w_{i}(t \Delta)-w_{i}((t-1) \Delta)=-\sum_{j=1}^{n} Q_{i j}^{t} y_{i j}^{t} .
$$

Relying on this discrete Lanchester's law, the continuous model is approximated discretely by modifica- tion of the last two assumptions:

$A_{2}^{\prime}$ : The offensive firepower of each weapon and hence the strategies $x$ and $y$ remain constant over a minimal period of length $\Delta$.

$A_{3}{ }^{\prime}$ : The decision-making of both players is performed at the beginning of each of $\mathrm{N}$ periods $[0, \Delta],[\Delta$, $2 \Delta], \cdots,[(N-1) \Delta, N \Delta]$, where $T=N \Delta$. The changes in weapon system in a period are reflected in the decision-making of the next period.

From Assumptions $A_{1}, A_{2}^{\prime}$, and $A_{3}{ }^{\prime}$ and the discrete Lanchester's law (2), we obtain the following discrete model for defender:

$$
\begin{aligned}
& \min _{x^{1}} \max _{y^{1}} \min _{x^{2}} \max _{y^{2}} \cdots \min _{x^{N}} \max _{y^{y}} c^{T} x+d^{T} y, \\
& \text { s.t. } \sum_{j=1}^{n} x_{i j}^{t}=w_{i}^{t}, \quad \forall i \in\{1, \cdots, m\}, \quad \forall t \in[0, T], \\
& w_{i}^{t+1}=w_{i}^{t}-\sum_{j=1}^{n} Q_{i j}^{t} y_{i j}^{t}, \quad \forall i \in\{1, \cdots, m\}, \quad \forall t \in[0, T], \\
& \sum_{i=1}^{m} y_{i j}^{t}=z_{j}^{t}, \quad \forall j \in\{1, \cdots, n\}, \quad \forall t \in[0, T], \\
& z_{j}^{t+1}=z_{j}^{t}-\sum_{i=1}^{m} P_{i j}^{t} x_{i j}^{t}, \quad \forall j \in\{1, \cdots, n\}, \quad \forall t \in[0, T], \\
& x_{i j}^{t}, y_{i j}^{t} \geq 0, \quad \forall i, \forall j, \forall t \text {. }
\end{aligned}
$$

The payoffs are assumed to be determined by the survived weapons, for instance, the number of landing crafts survived to the end of war-game. The objective function is represented by $c^{T} x+d^{T} y$, therefore, the linear combination of strategy $x$ and $y$ from (2). By nature we assume the objectives of two parties are completely opposite, namely, zero-sum. Each player expects that the other player does his best to respond her decision. Although it is a general form to split min-max of each level is general form, it may safely be said that single period include min-max by assumption $A_{3}^{\prime}$.

As a result, the sequential simultaneous game of $\mathrm{N}$ period is re-modeled on $N$-level min-max problem with a defender's perspective. By replacing $\min _{x} \max _{y}$ of each level with $\max _{y} \min _{x}$ in the objective, in addition, we obtain the model for the attacker. One may well criticize this discrete model in that the changes are reflected and decisions are made in the beginning of each period. This pragmatic model however, as seen later, facilitates the solution method. Also, this model seems more realistic than the continuous model in some sense. Assumption $A_{3}$ perhaps describes best the loss of strength of a combat force. But, in the same time, it is unrealistic to assume that the human operation can execute the strategies that change in an infinitesimal time.

We will show in Section 3 that there is equilibrium of this zero-sum game by showing that for any linear function $c^{T} x+d^{T} y$, its $\min _{x} \max _{y}$ and $\max _{y} \min _{x}$ problem have the same optimal objective value. Then, it will 
imply that equilibrium is optimal to both players in the sense that the corresponding $x$ and $y$ are a pair of dominant strategies.

\section{EXISTENCE OF EQUILIBRIUM}

\subsection{Intractability of General Linear Min-Max Programs}

Theorem 3.1: Linear min-max programming is NP-hard (Hansen et al., 1992).

\subsection{Existence of Equilibrium of Our Model}

It is well known that every finite game has a Nash equilibrium (Nash, 1951). Here, by a finite game, we mean a game in which the number of players and the number of pure strategies that each of the players can command are both finite.

Furthermore, if, in addition, the payoffs of two players are directly opposite or zero-sum like ours, the existence of a Nash equilibrium, or a saddle point is established by its equivalence to the duality of linear programming. The min-max theorem of a finite zerosum game dubbed as matrix game is one of most celebrated result of early works of game theory (Dantzig, 1963; Gale, 1960) connecting equilibria of a game and optima of an optimization problem.

This particular equivalence implies that there is a minimum payoff that the defender can achieve regardless of the attacker's strategic choice. Symmetrically, there is a strategy of the attacker that guarantees a maximum payoff in spite of the offender's choice and the two payoffs are the same.

Thus, once we have shown that our model is a finite zero-sum game, a particular implication of this theory to our problems is that the Nash equilibria are unique up to their payoffs and hence they are optimal for both players. This uniqueness makes our war-game model particularly credible in its suggested warfare strategies or reinfor-cement of area defense system computed from it.

Proposition 3.2: The problem (3) has saddle points whose payoffs are unique.

Proof: In the light of the min-max theorem (Dantzig, 1963; Gale, 1960) relying on the duality of linear programming, it suffices to show that (4) is equivalent to a finite zero-sum game. Since the number of player is two and the payoffs are zero-sum, it only remains to show that any strategy of each player is the convex combination of a finite set of pure strategies.

A key observation is that $x$ and $y$-values are uniquely determined once we have decided the ratio of the units of weapon $i$ of the defender to firing at weapon $j$ of the attacker to the total units available at the beginning of the $t$-th period for each $t \in\{1,2, \cdots, N\}$ :

- $\sigma_{i j}^{t}$ : the ratio of the units of weapon $i$ of the defender to its total units available at the beginning of $t$-th period that fires at the weapon $j$ of the attacker during $t$ th period:

$$
\sigma_{i j}^{t}=\frac{x_{i j}^{t}}{\sum_{j=1}^{n} x_{i j}^{t}}, \forall i, \quad j, \text { and } t .
$$

- $\tau_{i j}^{t}$ : the ratio of the units of weapon $j$ of the attacker to its total units available at the beginning of $t$-th period that fires at the weapon $i$ of the defender during $t$-th period:

$$
\tau_{i j}^{t}=\frac{y_{i j}^{t}}{\sum_{i=1}^{m} y_{i j}^{t}}, \forall i, \quad j, \text { and } t .
$$

We denote the vector $\sigma$ of $\sigma_{i j}^{t}$ 's in their lexicographic order of $t, i$ and $j$ :

$$
\begin{gathered}
\sigma=(\underbrace{\sigma_{11}^{1}, \sigma_{12}^{1}, \cdots, \sigma_{1 n}^{1}}_{i=1, t=1}, \underbrace{\sigma_{i=2, t=1}}_{\sigma_{21}^{1}, \sigma_{22}^{1}, \cdots, \sigma_{2 n}^{1}} \cdots \underbrace{\sigma_{m 1}^{1}, \sigma_{m 2}^{1}, \cdots, \sigma_{m n}^{1},}_{i=1, t=2}, \underbrace{\sigma_{21}^{2}, \sigma_{22}^{2}, \cdots, \sigma_{2 n}^{2}}_{i=m, t=1}, \cdots \underbrace{\sigma_{m 1}^{2}, \sigma_{m 2}^{2}, \cdots, \sigma_{m n}^{2},}_{i=2, t=2} \\
\quad \underbrace{\sigma_{11}^{2}, \sigma_{12}^{2}, \cdots, \sigma_{11}^{2}}_{i=m, t=2}, \underbrace{\sigma_{11}^{N}, \sigma_{12}^{N}, \cdots, \sigma_{1 n}^{N}}_{i=1, t=N}, \underbrace{\sigma_{21}^{N}, \sigma_{22}^{N}, \cdots, \sigma_{2 n}^{N}}_{i=2, t=N}, \cdots \underbrace{\sigma_{m 1}^{N}, \sigma_{m 2}^{N}, \cdots, \sigma_{m n}^{N}}_{i=m, t=N}) .
\end{gathered}
$$

Thus, each $\sigma$ represents a strategy of the defender if the following condition holds.

$$
S_{\text {def }}=\left\{\sigma: \sum_{j=1}^{n} \sigma_{i j}^{t}=1, \forall i, t, \sigma_{i j}^{t} \geq 0, \forall i, j, t\right\} .
$$
attacker,

We denote $\tau$ similarly and the strategy set of the

$$
S_{o f f}=\left\{\tau: \sum_{i=1}^{m} \tau_{i j}^{t}=1, \forall j, t, \tau_{i j}^{t} \geq 0, \forall i, j, t\right\} .
$$

It is easy to see that once $\sigma$ and $\tau$ have been decided, the payoffs of the two players are uniquely determined.

Furthermore, for each feasible solutions $(x, y)$ of $(3)$, there is a corresponding pair $(\sigma, \tau)$. Notice that when there is a weapon whose available units is zero, more than one pairs of $(\sigma, \tau)$ may correspond to the same $(x$, $y$ ). However, there payoffs are identical. Thus, the problem (3) is equivalent to the two-person zero-sum game with the strategy sets $S_{d e f}$ and $S_{\text {off }}$ of two players. 

fender.

Consider the following strategy subset of the de-

$$
P_{d e f}=\left\{\sigma: \sum_{j=1}^{n} \sigma_{i j}^{t}=1, \forall i, t, \sigma_{i j}^{t} \in\{0,1\}, \forall i, j, t\right\} .
$$

Then it is easy to see that $\left|P_{d e f}\right|=n^{m N}$. Similarly a strategy subset of the attacker, $P_{o f f}$, is defined. Then $\mid P_{o f f}$ $\mid=m^{n N}$.

Our claim is any strategy in $S_{\text {def }}$ is the convex combination of a set of strategies from $P_{\text {def. }}$. To see this, notice that $S_{d e f}$ from (4) is a linear program relaxation of $P_{\text {def }}$ from (5). Since coefficient matrix of the left hand side of (4) is totally unimodular and the right hand side of (4) is integer, $S_{d e f}$ is integral. In other words, every strategy in $S_{\text {def }}$ is a mixed strategy of the pure strategies from $P_{d e f}$.

More specifically, denoting by $p\left(j_{1}^{1}, \cdots, j_{m}^{1} ; j_{1}^{2}, \cdots\right.$, $\left.j_{m}^{2}, \cdots ; j_{1}^{N}, \cdots, j_{m}^{N}\right)$ the vector $\left(\sigma_{i j}^{t}\right) \in P_{d e f}$ such that for every $t, i \in\{1, \cdots, m\}$, and $j \in\{1, \cdots, n\}$,

$$
\sigma_{i j}^{t}=\left\{\begin{array}{l}
1, \text { if } \quad j=j_{i}^{t}, \\
0, \text { otherwise. }
\end{array}\right.
$$

Note that $p\left(j_{1}^{1}, \cdots, j_{m}^{1} ; j_{1}^{2}, \cdots, j_{m}^{2} ; \cdots ; j_{1}^{N}, \cdots, j_{m}^{N}\right)$ stands for the pure strategy that during $t$-th period the all available units of weapon $i$ of the defender are allocated to firing at weapon $j_{i}$ of the attacker.

Then, it is easy to see that, if $s_{d e f}=\sigma$, then

$$
s_{d e f}=\sum_{p \in S_{d e f}} \lambda_{p} p .
$$

where, for $p \equiv p\left(j_{1}^{1}, \cdots, j_{m}^{1}, j_{1}^{2}, \cdots, j_{m}^{2} ; \cdots ; j_{1}^{N}, \cdots, j_{m}^{N}\right)$,

$$
\lambda_{p}=\prod_{t=1}^{N} \prod_{i=1}^{m} \sigma_{i j_{i}^{t}}^{t} .
$$

Clearly, a symmetric argument applies to the case of the attacker.

\section{CONCLUSION AND OPEN PROBLEMS}

Even with the accurate information of military power of both ally and enemy, it is difficult to figure out the reasonable level of current weapon system in specific area for guerrilla defense and optimal strategy in battle.

This paper reaches to conclusion of a military strategy in optimal point of view. We proposed a mathematical war-game model that describes a raid in which the maneuver is relatively certain. As a result, we found out that this mathematical model is a multi-weapon extention of the Lanchester's law. Furthermore, we proved existence of equilibrium of the model by a strategy, which is applied to both ally and enemy, can be expressed in linear combination of pure strategies. The existence of optimal strategies for both players of the problem (3) motivates a conjecture that the existence of optimal strategies for continuous zero-sum multi-weapon model.

An efficient algorithm for war-game model based on discrete time needs to be studied. A gap occurs between continuous time war-game model and discrete one also needs to be studied.

\section{REFERENCES}

Colegrave, R. K. and J. M. Hyde, "The Lanchester square-law model extended to a $(2,2)$ conflict," IMA $J$ Appl Math 51 (1993), 95-109.

Dantzig, G. B., Linear Programming and Extentions, Princeton University Press, 1963.

Gale, D., The Theory of Linear Economic Models, McGraw-Hill, New York, 1960.

Hsiao, H. and S. M. Guu, "A differential game for airland combat operation," WSEAS Transactions on systems 4 (2004), 1535-1542.

Hansen, P., B. Jaumard, and G. Savardb, "New branchand-bound rules for linear bilevel programming," SIAM J Sci Stat Comput 13 (1992), 1194-1217.

Im, J. S., Mathematical model for amphibious raid wargame, M. S. thesis, Seoul National University, 2012.

Kaup, G. T., D. J. Kaup, and N. M. Finkelstein, "The Lanchester (n, 1) problem," J Oper Res Soc 56 (2005), 1399-1407.

Kawara, Y., "An allocation problem of support fire in combat as a differential game," Oper Res 21 (1973), 942-951.

Lanchester, F. W., Aircraft in warfare: the dawn of the fourth arm, Constable and Co., London, England, 1916.

Marin, J. A., "A model for optimizing field artillery fire, M. S. thesis, Naval Postgraduate School, 1989.

Nash, J., "Non-cooperative games," Annals of Mathematics 54 (1951), 286-295.

Roberts, D. M. and B. W. Conolly, "An extention of the Lanchester square law to inhomogeneous forces with an application to force allocation methodology," J Oper Res Soc 43 (1992), 741-752.

Taylor, J. G., "Target selection in Lanchester combat: linear-law attrition process," Naval Res Logist Q 20 (1973), 673-697.

Taylor, J. G., "Solving Lanchester-type equations for 'Modern Warfare' with variable coefficients," Oper Res 22 (1974a), 756-770.

Taylor, J. G., "Target selection in Lanchester combat: heterogeneous forces and time-dependent attrition- 
rate coefficient," Naval Res Logist Q 21 (1974b), 683-704.

Taylor, J. G. and S. H. Parry, "Force-ratio considerations for some Lanchester-type models of warfare,"
Oper Res 23 (1975), 522-533.

Welr, D. M., Naval gunfire support of amphibious operations: past, present, and future, Naval Surface Weapons Center, 1977. 